\title{
Robust mechanisms of ventral furrow invagination require the combination of cellular shape changes
}

\author{
Vito Conte ${ }^{1}$, José J Muñoz ${ }^{2}$, Buzz Baum ${ }^{3}$ and Mark Miodownik ${ }^{1}$ \\ ${ }^{1}$ Materials Research Group, Division of Engineering, King's College London, UK \\ ${ }^{2}$ Department of Applied Mathematics III, University Polit. Catalonia, Barcelona, Spain \\ ${ }^{3}$ MRC Laboratory for Molecular Cell Biology, University College London, UK \\ E-mail: mark.miodownik@kcl.ac.uk
}

\begin{abstract}
Ventral furrow formation in Drosophila is the first large-scale morphogenetic movement during the life of the embryo, and is driven by co-ordinated changes in the shape of individual epithelial cells within the cellular blastoderm. Although many of the genes involved have been identified, the details of the mechanical processes that convert local changes in gene expression into whole-scale changes in embryonic form remain to be fully understood. Biologists have identified two main cell deformation modes responsible for ventral furrow invagination: constriction of the apical ends of the cells (apical wedging) and deformation along their apical-basal axes (radial lengthening/ shortening). In this work, we used a computer 2D finite element model of ventral furrow formation to investigate the ability of different combinations of three plausible elementary active cell shape changes to bring about epithelial invagination: ectodermal apical-basal shortening, mesodermal apical-basal lengthening/shortening and mesodermal apical constriction. We undertook a systems analysis of the biomechanical system, which revealed many different combinations of active forces

(invagination mechanisms) were able to generate a ventral furrow. Two important general features were revealed. First that combinations of shape changes are the most robust to environmental and mutational perturbation, in particular those combining ectodermal pushing and mesodermal wedging. Second, that ectodermal pushing plays a big part in all of the robust mechanisms (mesodermal forces alone do not close the furrow), and this provides evidence that it may be an important element in the mechanics of invagination in Drosophila.
\end{abstract}

(Some figures in this article are in colour only in the electronic version)

\section{Introduction}

In recent decades, studies of animal morphogenesis have focused on the way in which genetic information guides development. This has led to the identification of genes regulating key aspects of gastrulation movements and to detailed descriptions of the dynamics of cell and tissue movements that accompany these major changes in tissue form in wild type and mutant animals (Keller et al 2003). Importantly, these studies have identified common processes that appear to play critical roles during morphogenesis in all Metazoa. These include the infolding of twodimensional sheets of epithelial cells that make up the early embryonic epithelium to generate endoderm, mesoderm and the neural tube in organisms as diverse as Caenorhabditis elegans, Drosophila melanogaster, sea urchins, amphibians and mammals (Lecuit and Lenne 2007).

It is possible that the widespread use of epithelial invagination as a morphogenetic principle in Metazoa is a simple reflection of common ancestry and of the evolutionary conservation of morphogenetic regulators such as E-Cadherin, non-muscle Myosin II and Snail family proteins. 


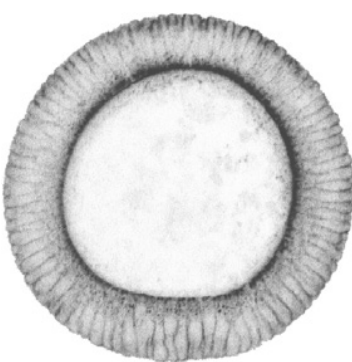

(a)

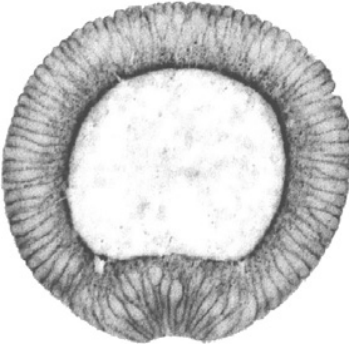

(b)

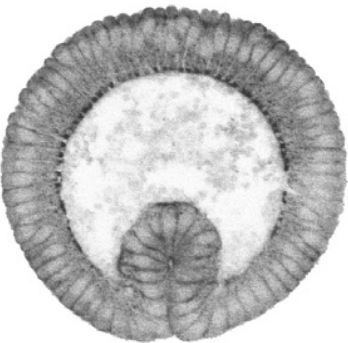

(c)

Figure 1. Three successive stages of the ventral furrow invagination in the Drosophila melanogaster embryo (Muñoz et al 2007). Cells are outlined using phalloidin to highlight filamentous actin (dorsal is up): (a) initial state; $(b)$ ventral cells have constricted their apical surfaces and elongated radially; $(c)$ ventral cells have invaginated and the furrow has formed.

Alternatively, the ubiquity of this type of morphogenetic movement could reflect generic physical constraints that limit the types of cell and tissue movements that are possible within a developmental context and/or the types of mechanical regimen that can be used to bring about large-scale changes in tissue organization. To address this question, we have taken a mechanical modelling approach; our central argument being that it is only by comparing modelling with experiment that such biomechanical processes can be properly deciphered. In this, we have been inspired by previous pioneering works in the field (Odell et al 1981, Clausi and Brodland 1993, Brodland and Clausi 1994, Davidson et al 1995, Taber 2008).

We have chosen ventral furrow formation in Drosophila as a model system for this analysis for two reasons. First, it is one of the best-studied morphogenetic processes in biology. Second, ventral furrow formation is a comparatively simple but important mechanical process which, as the first largescale morphogenetic movement in the fly embryo, begins as soon as cellularization is complete. At this stage, the Drosophila embryo is composed of a relatively uniform single layer of columnar epithelial cells, surrounded by a semi-hard shell composed of a rigid chorion and a vitelline membrane, which contains an incompressible liquid, the yolk. This organization can be readily appreciated in crosssection, where the epithelial blastoderm can be seen to form a circular array of columnar cells with their apical-basal axes regularly aligned along the axis of radial symmetry, with the apical surfaces facing outwards (figure 1). Each cell maintains lateral contacts with its two neighbouring epithelial cells. Then, over a period of $\sim 15 \mathrm{~min}$, a single morphogenetic movement transforms this simple topology into a multilayered structure by inducing the internalization of cells most ventrally positioned in the embryonic epithelium. Successive mesenchymal transformations give rise to the mesoderm.

As with other morphogenetic movements in animal development, ventral furrow formation is thought to be driven by coordinated changes in the shape of individual cells at the site of active movement, which generate global changes in tissue organization (Costa et al 1993, Keller et al 2003, Leptin and Grunewald 1990, Leptin 1999). The first observable event in this process is the flattening of the apical surfaces of cells within the most ventral region of the cellular blastoderm (Sweeton et al 1991, Leptin and Grunewald 1990). This is followed by the constriction of the apical domain of scattered cells within this population. As these stochastic apical constriction events become more widespread, it is thought that coordinated changes in individual cell shape translate into formation of a shallow indentation along the ventral surface of the embryo (Odell et al 1981). At the same time, cells within the furrow lengthen along their apical-basal axes, reaching up to 1.7 times their original height (Costa et al 1993). Once the furrow has formed, its constituent cells then begin to shorten back to their original length, whilst keeping their apical ends constricted (Costa et al 1993), resulting in their assuming a wedge-like form (basal expansion). This second change in cell shape has been proposed to constitute the final push that drives furrow internalization (Costa et al 1993, Leptin 1995). Additionally, it has been suggested by a number of authors that the lateral and dorsal ectodermal cells could help to drive ventral furrow invagination by pushing laterally on the sides of the prospective mesoderm; facilitating inward buckling and reinforcing internalization of the ventral furrow (Costa et al 1993, Leptin 1995, Muñoz et al 2007, Conte et al 2008).

The site at which the ventral furrow is formed is determined by two ventrally expressed transcription factors, Twist and Snail (Leptin 1995). Evidence to support this comes from genetic studies which have demonstrated: (i) that none of the morphogenetic events that accompany ventral furrow formation occur in the absence of Twist and Snail, and that (ii) the co-expression of Twist and Snail is sufficient to generate ectopic furrows (Roth 2004). How then are these effects of Twist and Snail achieved?

A combination of local Twist-induced expression of mesodermal genes and Snail-mediated repression of ectodermal cell fate therefore appears to bring about ventral furrow invagination, via cell biological events such as apical flattening, apical constriction (early wedging), early apical-basal lengthening, late apical-basal shortening and basal expansion (late wedging), as summarized in figure 2 . Nevertheless, whilst the molecular events underpinning apical constriction (the result of contraction of an actin-Myosin II ring tethered at adherens junctions) and the signalling pathways leading to its induction (via Fog and T48, upstream of RhoGEF2 and RhoA) are reasonably well understood, the other forces likely to be involved in furrow internalization are not. Importantly, it is not known which of the other changes in cell shape observed in wild-type ventral furrow formation 


\begin{tabular}{|c|c|c|c|c|c|c|c|c|}
\hline DROSOPHILA & TWIST & SNAIL & $\begin{array}{c}\text { apical } \\
\text { flattening }\end{array}$ & $\begin{array}{c}\text { apical } \\
\text { constriction }\end{array}$ & \begin{tabular}{|l} 
apical-basal \\
lengthening
\end{tabular} & \begin{tabular}{|c} 
apical-basal \\
shortening
\end{tabular} & $\begin{array}{c}\text { basal } \\
\text { expansion }\end{array}$ & INVAGINATION \\
\hline wild type & $\checkmark$ & $\checkmark$ & $\checkmark$ & $\checkmark$ & $\checkmark$ & $\checkmark$ & $\checkmark$ & $\checkmark$ \\
\hline snail mutants & $\checkmark$ & $x$ & $\checkmark$ & $x$ & $x$ & $\checkmark$ & $x$ & $x$ \\
\hline twist mutants & $x$ & $\checkmark$ & $x$ & $x$ & $\checkmark$ & $x$ & $x$ & $x$ \\
\hline $\begin{array}{c}\text { twist - snail } \\
\text { mutants }\end{array}$ & $x$ & $x$ & $x$ & $x$ & $x$ & $x$ & $x$ & $x$ \\
\hline
\end{tabular}

Figure 2. Genetic control of Twist and Snail expressions: the symbols $\sqrt{ } / \times$ denote the activation/repression of the corresponding gene, the presence/absence of the corresponding cell shape change in the midventral region of the mesodermal primordium or the success/failure of ventral furrow invagination.

are active processes and which reflect the passive response of physically connected tissue.

In order to examine how individual changes in cell shape contribute to the physical process of ventral furrow formation, we discretize a two-dimensional section of the embryo by resorting to finite elements methods (FEM). In recent years, this type of approach has been successfully applied to multicellular animal morphogenesis (Clausi and Brodland 1993, Brodland and Clausi 1994), in the study of sea urchin gastrulation (Davidson et al 1995) and epithelial invagination (Taber 2008, Chen and Brodland 2008, Ramasubramanian and Taber 2008). An interesting exception to these methodology is the work of Pouille and Farge (2008), where invagination is modelled by solely resorting to hydrodynamical equilibrium while increasing the apical membrane tension.

We have recently developed a 2D and 3D FEM model of ventral furrow formation in Drosophila melanogaster (Muñoz et al 2007, Conte et al 2008). This analysis showed that apical constriction within the ventral domain alone is not sufficient to drive internalization of the furrow, and it revealed the potential importance of apical-basal shortening outside of the ventral domain in furrow formation. However, the model simulated only one possible combination of cellular active forces. In the present work, we have extended the model and developed a new set of tools which allowed a systematic investigation of the effects of different combinations of active cell shape changes on furrow formation in wild type and mutant Drosophila embryos. From the exploration of the whole set of numerical simulations, it is then possible to test the relative robustness of different modes of invagination to perturbation.

\section{Methods}

In this work, we model the effect of both passive and active shape changes of individual cells on the global geometry of an epithelial blastoderm by resorting to the finite element method. The approach involves modelling the initial geometry of a 2D section of the embryo including the epithelial layer formed prior to invagination, the yolk, and vitelline membrane. We simulate different invagination mechanisms by decomposing the deformation at each time and at each point into an active and a passive part, as commonly employed in remodelling processes (Rodriguez et al 1994). Active shape changes are imposed on the epithelium at different points and at different times. The elasticity of the epithelium, together with the constraints, such as cells remaining in contact with each other and the presence of the vitelline layer and the yolk, then define a set of passive elastic forces that are required to keep the embryo in mechanical equilibrium. It is the combination of these mechanically necessary passive cell shape changes with the imposed active cell shape changes that determines the global shape change of the embryo. The mathematical description and our implementation can be found in detail in our other publications (Muñoz et al 2007, Conte et al 2008). Here we include only a basic description to enable a critical analysis of our approach and also to define the key parameters of the model.

\subsection{Modelling tissue deformation of the embryo}

The living tissue constituting the epithelium is modelled by resorting to a deformation gradient decomposition method, this means that the shape changes can be split into those due to active deformation and those due to passive deformation. According to this assumption, the total deformation gradient F, accounting for the finite deformations of the epithelium, is assumed to be the multiplicative composition of active $\left(\mathbf{F}_{a}\right)$ and passive $\left(\mathbf{F}_{e}\right)$ parts, that is, it has the form $\mathbf{F}=\mathbf{F}_{e} \mathbf{F}_{a}$ (Rodriguez et al 1994). Morphogenetic deformations of the epithelium are, therefore, conceived as the composition of active deformations determined by changes in cytoskeleton of cells $\left(\mathbf{F}_{a}\right)$ and finite passive elastic deformations of cells in response to the shape changes of all other parts of the embryo. In other words, the shape change of any finite part of the embryo is composed of a contribution due to its own active processes and a passive contribution due to the sum of shape changes throughout the embryo. However, there are also constraints imposed on the system: active shape changes determined by $\mathbf{F}_{a}$ alter the microscopical structure of the tissue and may lead to the appearance of material incompatibilities like tissue lacerations (i.e. discontinuity of 
(a)

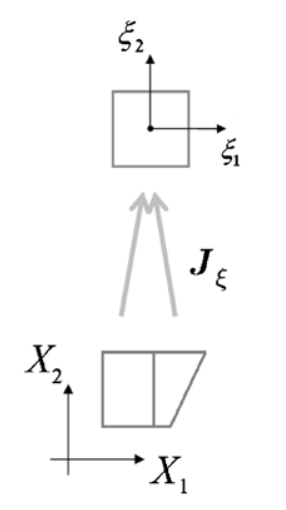

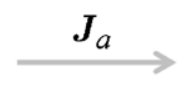

(b)
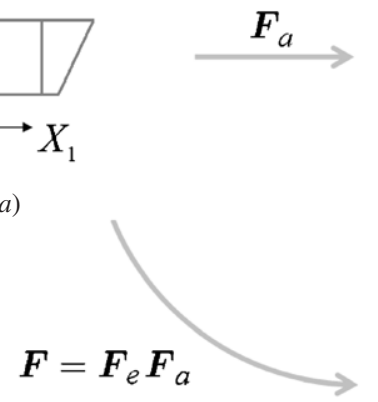
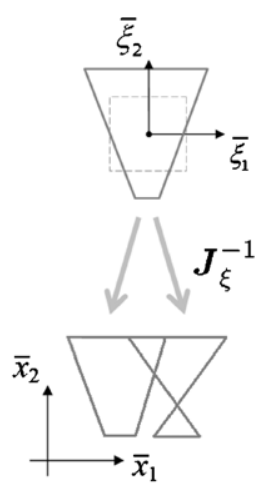

(c)

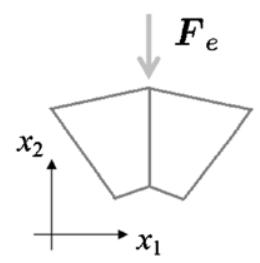

Figure 3. Scheme of the applied active deformation gradient $\mathbf{J}_{a}$ and other related tangent maps within the finite element context.

(a) Two adjacent finite elements in the initial configuration.

(b) Elemental active deformation of the common parent domain.

(c) Potential material incompatibilities.

$\mathbf{F}_{a}$ ), mutual co-penetrations between different material parts (overlapping), which may imply the violation of the condition $\operatorname{det} \mathbf{F}_{a}>0$. Since the elastic part $\mathbf{F}_{e}$ must satisfy the condition $\operatorname{det} \mathbf{F}_{e}>0$, at least within each finite element, and no material incompatibilities are observed during invagination in living tissues (i.e. the displacements are continuous and $\operatorname{det} \mathbf{F}>0$ ), $\mathbf{F}_{a}$ and $\mathbf{F}_{e}$ are modelled as piecewise continuous, the latter being responsible for the continuity of the epithelium. Figure 3 illustrates this kinematic decomposition in terms of the tangent maps $\left(\mathbf{F}, \mathbf{F}_{a}\right.$ and $\mathbf{F}_{e}$ ) for the active wedging and elongation of two adjacent finite elements (cells), and shows how the elastic deformation accounts for the compatibility of the global morphogenetic deformation.

Mechanical equilibrium, and therefore $\mathbf{F}_{e}$, is obtained by minimizing the integral of the elastic energy function $\Psi\left(\mathbf{F}_{e}\right)$ in the whole domain of the embryo (Muñoz et al 2007). The material is assumed to be (compressible) hyperelastic and homogeneous throughout the embryo, with an energy function given by $\Psi\left(\mathbf{F}_{e}\right)=\frac{\lambda}{2} \ln \left(\operatorname{det} \mathbf{F}_{e}\right)^{2}+\frac{\mu}{2}\left(\operatorname{tr}\left(\mathbf{F}_{e}^{T} \mathbf{F}_{e}\right)-3\right)-$ $\mu \ln \left(\operatorname{det} \mathbf{F}_{e}\right)$. We have chosen the approximated values $\lambda=577 \mathrm{~N} \mathrm{~m}^{-2}$ and $\mu=384 \mathrm{~N} \mathrm{~m}^{-2}$, which correspond to those extracted from the analysis on the epithelium of a sea urchin (Davidson et al 1999). Nevertheless, our model is kinematically driven by $\mathbf{F}_{a}$, which is independent of $\lambda$ and $\mu$ (see the analytical expressions in equations (1) and (2)); accordingly, our results and conclusions are independent of the values of $\lambda$ and $\mu$, although the final stress level will be dependent on the material constants (Muñoz et al 2007).

\subsection{Constructing a $2 D$ model of the embryo}

There are some aspects of invagination which can only be modelled by using a fully 3D model, as shown in Conte et al
(2008). However, for the purposes of this analysis, we have constructed a circular 2D model that closely approximates 2Dcross sections of both a 3D model and the living Drosophila embryo (data not shown). For the sake of simplicity, the epithelial layer is modelled as having an initial uniform thickness, so that all cells are of the same size (although, individual cells are not explicitly modelled). The inner region of the embryo, the yolk, is modelled as zero-volume variation constraint rather than as a material. We then divided up the epithelial tissue into two regions: the prospective mesoderm (which we will simply refer to as 'mesoderm') and the ectoderm. Active shape changes are independently applied to the two domains. Figure 4 shows a schematic of the embryo model and the mapping involved.

\subsection{Parametric active shape change}

Cells in this system undergo active shape changes driven by internal processes. Each finite element in the ventral region of the discretized geometry is representative of a mesodermal cell; all finite elements in our model are subjected to a single or a combination of the two following active shape changes: apical constriction (wedging), and radial (apicalbasal) lengthening or shortening, as illustrated in figure 4 .

We apply those active deformations onto the parent domain common to each finite element which is described by the parametric coordinates $\xi \equiv\left(\xi_{1}, \xi_{2}, \xi_{3}\right),-1 \leqslant \xi_{i} \leqslant+1$. The active deformation is thus defined through the tangent map $\mathbf{J}_{a}$, which is in turn decomposed into an elongation component $\left(\mathbf{J}_{e l}\right)$ and an apical constriction component $\left(\mathbf{J}_{a c}\right)$ (see figure 3 ). The analytic expressions of the two elementary transformations $\mathbf{J}_{a c}$ and $\mathbf{J}_{e l}$ are given by

$$
\begin{gathered}
\mathbf{J}_{a c}(\tau)=\left[\begin{array}{ccc}
1+\xi_{2} \hat{\omega}_{a c}(\tau) & \xi_{1} \hat{\omega}_{a c}(\tau) & 0 \\
0 & 1 & 0 \\
0 & 0 & 1
\end{array}\right], \\
\mathbf{J}_{e l}(\tau)=\left[\begin{array}{ccc}
1+\hat{\omega}_{e l}(\tau) & 0 & 0 \\
0 & \frac{1}{1+\hat{\omega}_{e l}(\tau)} & 0 \\
0 & 0 & 1
\end{array}\right] .
\end{gathered}
$$

These two matrices are the essence of the model as they represent active wedging and the active lengthening/ shortening (along the radial, apical-basal axes) of epithelial cells, respectively. These expressions are written in terms of the two functional quantities $\hat{\omega}_{a c}(\tau)$ and $\hat{\omega}_{e l}(\tau)$, which are two parameters used to implement the intensity of each active deformation in function of a parameter $\tau$ (Muñoz et al 2007), which we call pseudo-time.

We model invagination by applying an appropriate active shape factor to the ectoderm (factors $\hat{\omega}_{a c}^{E}$ and $\hat{\omega}_{e l}^{E}$ ) and to the mesoderm (factors $\hat{\omega}_{a c}^{M}$ and $\hat{\omega}_{e l}^{M}$ ). We assume that $\hat{\omega}_{a c}^{E}=0$ in all cases, since there is no in vivo evidence for apical constriction in the ectodermal primordium during invagination. Accordingly, deformations in each simulation can be fully characterized through the vector $\hat{\omega}(\tau) \equiv$ $\left(\hat{\omega}_{e l}^{E}(\tau), \hat{\omega}_{e l}^{M}(\tau), \hat{\omega}_{a c}^{M}(\tau)\right)$, which will also be referred to as the $\omega$ vector. This vectorial quantity describes the set of active deformations imposed on the embryo's geometry. As 


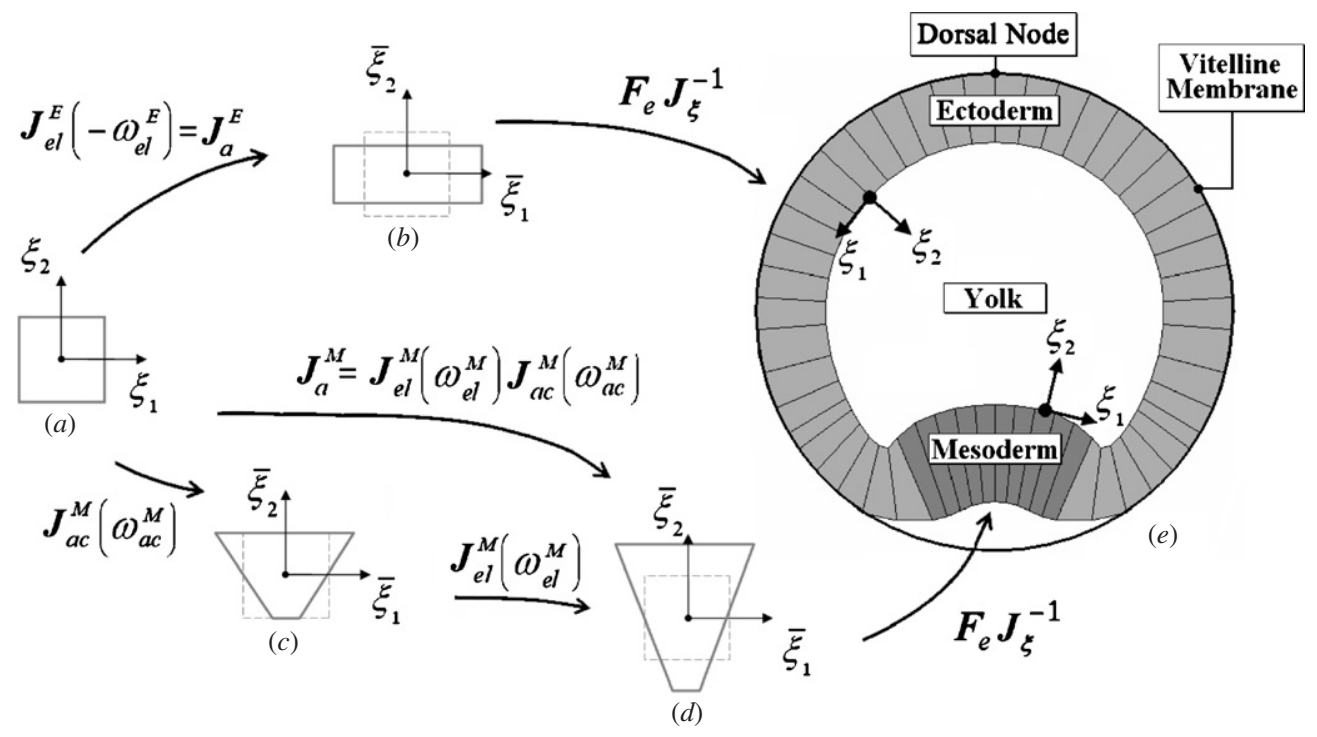

Figure 4. A schematic of the model showing the vitelline membrane, yolk, ectoderm, mesoderm and how the active deformations $\omega_{e l}^{E}, \omega_{e l}^{M}, \omega_{a c}^{M}$ map onto the finite element geometry (see section 2). The active deformation $\mathbf{J}_{a}$ is applied to the parent domain of all the finite elements $(a)$, through the tangent maps $\mathbf{J}_{a}^{E}$ (ectoderm) and $\mathbf{J}_{a}^{M}$ (mesoderm). The parent domain of the elements in the ectoderm is subjected to active shortening $\mathbf{J}_{e l}^{E}(b)$. The parent domain of the elements in the mesoderm is subjected to active radial lengthening $\mathbf{J}_{a c}^{M}(c)$ followed by active wedging $\mathbf{J}_{e l}^{M}$, in order to produce the final active deformation $(d)$. The final deformed geometry, as a result of mapping the actively deformed parent domain onto the current element (tangent $\operatorname{map} \mathbf{J}_{\xi}^{-1}$ ) and performing elastic equilibrium (tangent map $\mathbf{F}_{e}$ ) is depicted in (e). Here, motion of dorsal node was fully constrained in order to prevent undesired free-body motions of the whole epithelium.

expected, from the assumption of elasticity (and as numerically confirmed, see figure 8), the deformed configuration of the embryo is independent of the analytic form of the functions $\hat{\omega}_{e l}^{E}, \hat{\omega}_{e l}^{M}$ and $\hat{\omega}_{a c}^{M}$ in the simulation's interval $\left[0, \tau_{\max }\right]$, once the values $\hat{\omega}(0)$ and $\hat{\omega}\left(\tau_{\max }\right)$ are prescribed. In other words, the phenotypical deformations obtained at each value of $\tau$ are path-independent. For this reason, the components of the active shape load vector will be simply assumed to be linear (constant slope), and therefore with the form

$$
\hat{\omega}(\tau)=\tau \omega_{a},
$$

where

$$
\omega_{a} \equiv\left(\omega_{e l}^{E}, \omega_{e l}^{M}, \omega_{a c}^{M}\right),
$$

will be referred to as the active shape vector.

The set of all phenotypes achievable with our model corresponds to the $3 \mathrm{D}$ real-space $\mathbb{R}^{3}$ with Cartesian coordinates $\left(\omega_{e l}^{E}, \omega_{e l}^{M}, \omega_{a c}^{M}\right)$, which will be simply referred to as the $\omega$ space. Each curve $\hat{\boldsymbol{\omega}}(\tau)$ in this space identifies a numerical simulation performable with our model. The path-independence property ensures that at each point of the $\boldsymbol{\omega}$ space, there is a corresponding phenotype which is independent of the path $\hat{\omega}(\tau)$ followed to reach this point. Consequently, the vector $\boldsymbol{\omega}_{a}$ characterizes the shape given to the geometry by $\hat{\boldsymbol{\omega}}_{a}$ (see equation (3)), and so does every other vector parallel to $\boldsymbol{\omega}_{a}$. Therefore, without loss of generality, it will be assumed that $\left\|\omega_{a}\right\|=1$ and, accordingly, that $\hat{\boldsymbol{\omega}}_{a}$ is representable by the unit vector $\boldsymbol{\omega}_{a}$ (orientation) and the scalar $\tau$ (magnitude). In this way, the set of all possible simulations performable with our model can be graphically represented in the $\boldsymbol{\omega}$ space through the surface of the unit-sphere of Cartesian equation $\left\|\boldsymbol{\omega}_{a}\right\|=1$, which will be simply referred to as the $\boldsymbol{\omega}$ sphere (see figure 5). On this sphere, node A represents simulations with

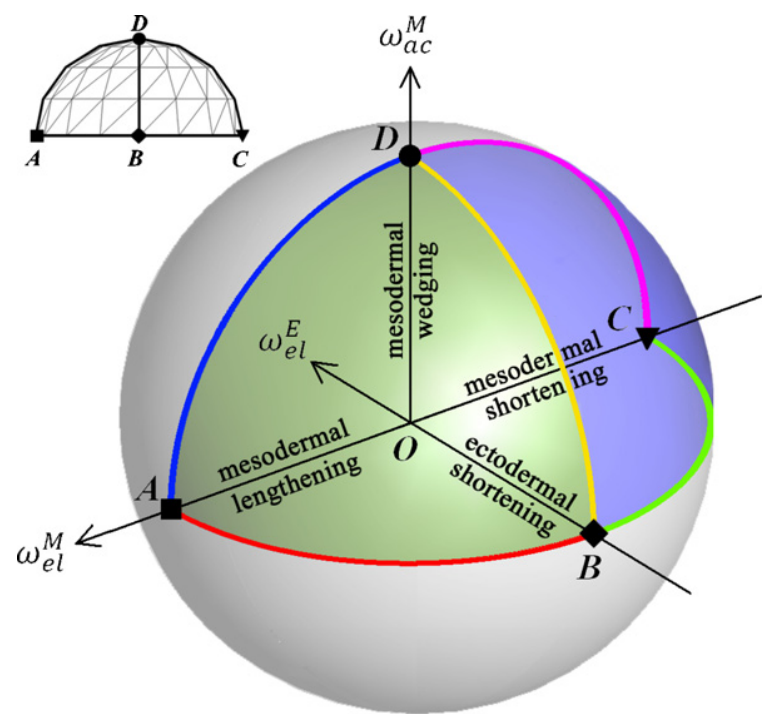

Figure 5. A graphical description of $\omega$ space: simulations with vectors $\omega_{a}$ corresponding to grey regions of the unit sphere generate deformations incompatible with the invagination of the ventral furrow. The spherical sector $\Upsilon$ is highlighted in colour, and different colours correspond to different invagination mechanisms. Node A represents pure mesodermal lengthening with no other active shape changes present; node $\mathrm{C}$ represents pure mesodermal shortening; node B represents pure ectodermal shortening; node D represents pure mesodermal wedging. Points along the $\operatorname{arc} \widehat{\mathrm{AD}}$ represent combinations of mesodermal wedging and mesodermal lengthening. Points on the surface of the sector ABD represent combinations of mesodermal wedging, mesodermal lengthening and ectodermal shortening, etc.

pure mesodermal shortening and no other active shape change is present; node $\mathrm{C}$ represents pure mesodermal lengthening; 
node $\mathrm{B}$ represents pure ectodermal shortening; node D represents pure mesodermal wedging. Thus points along the arc $\overparen{\mathrm{AD}}$ represent combinations of mesodermal wedging and mesodermal lengthening, while points on the surface of the sector ABD represent combinations of mesodermal wedging, mesodermal lengthening and ectodermal shortening, and so on. The analysis of the simulations corresponding to all points on the $\boldsymbol{\omega}$ sphere allows us to explore the whole $\boldsymbol{\omega}$ space, i.e. the full set of combinations of active shape changes in the model.

\subsection{Implementing constraints and contact}

The internal yolk of the embryo is nearly incompressible and exerts a pressure on the epithelial tissue that plays an important role in invagination (Muñoz et al 2007, Conte et al 2008). This is modelled by enforcing the yolk to maintain a constant area. Numerically this is achieved by using a penalty method, where the total elastic potential of the epithelium is complemented by an extra potential. This method is computationally efficient in that it ensures minimal variation of the yolk area whilst not requiring the numerical complexities of treating the yolk as a fluid.

We model the vitelline membrane as a rigid external shell that surrounds the 2D embryo (figure 4); this circle behaves as a rigid body with respect to the inertial reference system of the model. The contact between the cells and this shell is assumed frictionless, and is modelled resorting to the master-slave approach (Muñoz and Jelenić 2004), which circumvents the use of Lagrange multipliers or penalty methods. Additionally, the rigid shell has been interpolated with smooth $\left(C^{1}\right)$ BSplines (Muñoz 2008). In this way, we recover the quadratic convergence of the iterative Newton-Raphson process, which represents a significant improvement over the methods used in our previous model (Muñoz et al 2007).

\subsection{Detecting invagination}

The kinematics of ventral invagination can be characterized by two basic observations: inward bending of the epithelial tissue, internally towards the region occupied by the yolk, and the constancy of the epithelium topology until two opposite sides of the invaginated furrow meet one another (self-contact). At this stage, a topological transition is induced in the epithelial layer, and cells initially far apart in the epithelium extend their neighbourhood coordination by joining cells from other parts of the epithelium. In agreement with the two experimental observations described, the numerical simulations were classified in the following two sets:

(1) Successful invagination: the following three events occurred:

(a) Inward bending of mesodermal epithelium.

(b) The elastic deformations do not violate the impenetrability condition of the matter, i.e. $\operatorname{det} \mathbf{F}>0$.

(c) Self-contact of the ectoderm at two initially distant points.

(2) Uncompleted or disrupted invagination: at least one of the following events was encountered: (a) Outward invagination occurs at some point of the epithelium.

(b) The elastic deformations violated the impenetrability condition of the matter, i.e. $\operatorname{det} \mathbf{F} \leqslant 0$.

(c) Further increase of the intensity $\tau$ did not lead to further shape changes.

The mechanical evolution was halted when a numerical simulation could be classified in one of the two sets. The value of the pseudo-time $\tau$ at this point is denoted by $\tau_{\max }$.

\section{Results}

Figure 6 shows an example of the model simulating a combination of ectodermal shortening coupled with wedging and radial lengthening of the mesodermal elements. As $\tau$ increases, the values of the vector $\hat{\omega}(\tau)$ vary until a successful invagination event takes place.

Figure 7 summarizes the phenotypical effects of changing the time evolution of the three active shape factors relatively to each other while keeping the final $\boldsymbol{\omega}_{a}$ constant. The middle vertical path corresponds to the simulation shown in figures $6(a)-(e)$. The other paths illustrate the impact of delaying/anticipating the application of a single active shape factor with respect to remaining shape changes. The two left-most paths (see figures $7(a)-(b)$ ) show, respectively, the situation when active radial shortening in the ectoderm $\hat{\omega}_{e l}^{E}(\tau)$ (i.e. ectodermal pushing) is delayed until near the end of the invagination process (see $\bar{\omega}_{e l}^{E}(\tau)$ in figure 8), and the case in which this forces anticipates mesodermal forces (see $\tilde{\omega}_{e l}^{E}(\tau)$ in figure 8). The two paths directly to the left and to the right of the central constant rate path (see figures $7(c)-(e)$ ) show the effects of similar manipulations on the active radial lengthening in the mesoderm $\hat{\omega}_{e l}^{M}(\tau)$. In the same way, the two left-most paths (see figures $7(f)-(g)$ ) show the effects of delaying/anticipating the active apical constriction $\hat{\omega}_{a c}^{M}(\tau)$ within the prospective mesodermal domain.

The effect of either delaying or anticipating the onset of a specific active shape change relative to the timing of the others can be assessed and visualized in quantitative terms by monitoring the progress of invagination through a quantitative measure of the height $h$ of the furrow, as shown in figure 8 . The figure demonstrates that regardless of the timing path, the final furrow height is identical. Furthermore, we can confirm that the whole phenotype is identical, and that this property held for all values of $\boldsymbol{\omega}_{a}$ we tested. In other words, the model exhibits path independence. It should be stressed that this is an entirely expected property for conservative mechanical systems, (like that explored here) under small strains and displacements, and not a curious property of invagination. In our case, we can state that neither the boundary conditions nor the large deformations violate this basic property of an elastic system.

The existence of path independence in the model enabled us to carry out a system analysis by exploring the phase space defined by the three active shape changes, and represented as the $\boldsymbol{\omega}$ sphere (see figure 5). The simulation shown in figure 6 represents one point on the $\omega$ sphere, a point on the sector $\mathrm{ABD}$ which connects the node $\mathrm{B}$ which represents pure ectodermal shortening, the node $\mathrm{D}$ which represents 


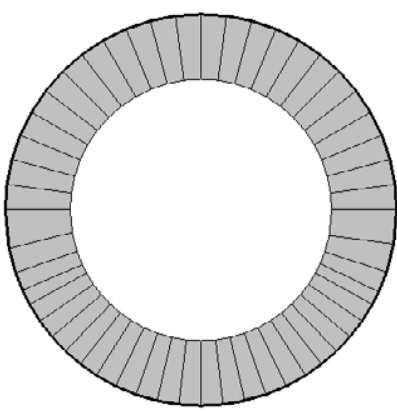

(a)

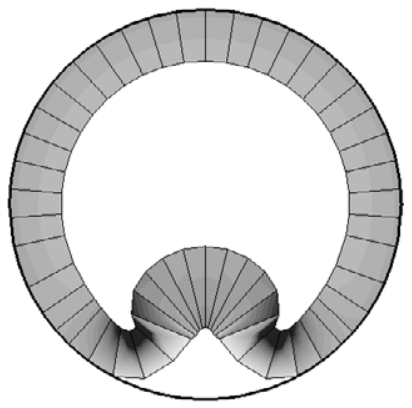

(d)

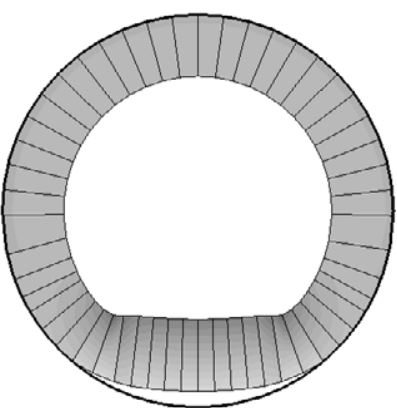

(b)

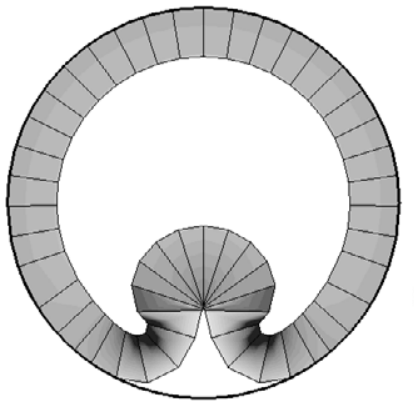

(e)

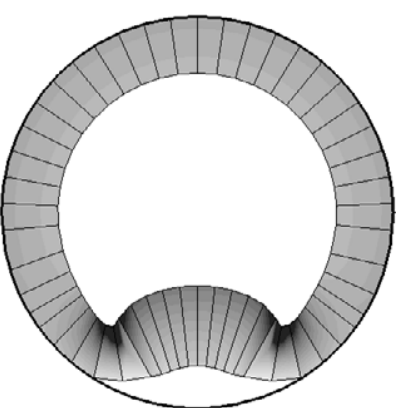

(c)

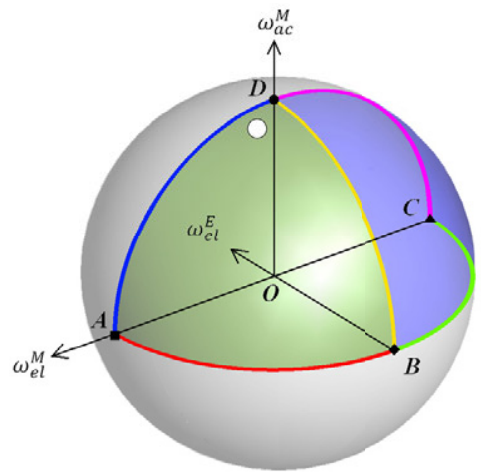

(f)

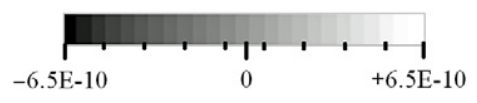

(g)

Figure 6. Successive stages of ventral furrow invagination for $\omega_{a} \equiv\left(\omega_{e l}^{E}, \omega_{e l}^{M}, \omega_{a c}^{M}\right)=(-0.24,0.24,0.94)$. (a) $\tau=0.000$ (initial state); (b) $\tau=0.500 ;(c) \tau=1.050 ;(d) \tau=1.500 ;(e) \tau=1.800 ;(f)$ location of mechanism in the $\omega$ space shown by white dot; $(g)$ key for the circumferential Cauchy stresses.

pure mesodermal wedging and node A which represents pure mesodermal lengthening. In order to fully explore the $\boldsymbol{\omega}$ space, we discretized the $\boldsymbol{\omega}$ sphere through a set of meridians and parallels both in the boreal $\left(\omega_{a c}^{M}>0\right)$ and austral $\left(\omega_{a c}^{M}<0\right)$ hemispheres. Simulations involving $\boldsymbol{\omega}$ vectors with components $\omega_{e l}^{E}>0$ (ectodermal radial lengthening) and $\omega_{a c}^{M}<0$ (mesodermal basal constriction) violate the necessary condition for ventral furrow invagination by resulting into phenotypes with outward bending of the ventral epithelium or with no closure of the furrow. Consequently, our inquiry about detection of possible ventral furrow invagination in the model was restricted to the spherical sector $\Upsilon \equiv\left\{\omega \in \mathbb{R}^{3} \mid \omega_{e l}^{E} \leqslant 0\right.$, $\left.\omega_{a c}^{M} \geqslant 0,\|\boldsymbol{\omega}\|=1\right\}$, as shown in figure 5. Within this spherical sector, we analysed a discrete set of simulations $\omega_{a} \in \Upsilon$ and we considered the corresponding phenotype at $\tau=\tau_{\max }$, when invagination was detected in accordance with the invaginationdetection criterion described in section 2.5. Figure 9 shows a map of the final phenotypes obtained. Here, phenotypes satisfying the definition of completed invaginated furrow are highlighted with white epithelium and those that fail are shown in light grey, whereas inner yolk is in dark grey.

It is interesting to note that there is only one shape change that can act on its own to cause invagination, namely ectodermal shortening (node B). All other single changes fail to cause invagination (although pure mesodermal wedging at node $\mathrm{D}$ comes close). All combinations of mesodermal lengthening/shortening and mesodermal wedging (paths along arcs $\overparen{\mathrm{CD}}$ and $\overparen{\mathrm{AD}}$ ) fail. As do most combinations of mesodermal lengthening/shortening and ectodermal shortening (paths along arcs $\overparen{\mathrm{AB}}$ and $\overparen{\mathrm{CB}}$ ). There is, however, a large region in which combinations of the three shape changes can cause successful invagination, albeit with distinctly different phenotypes. There are distinct phenotypic trends, such as that a greater component of mesodermal wedging causes a more spherical furrow, and a greater component of ectodermal shortening causes a more elongated furrow, see figure 9. In order to explore such phenotypic trends in a more quantitative fashion, we evaluated the height $h$ of the furrow. We then displayed such $h$ values as contour plots on the $\boldsymbol{\omega}$ sphere (see figure 10). The solid line on the plots represents the area within which successful invagination was obtained. This also corresponds to most, though not all, of the region with the highest furrows.

\section{Discussion}

The embryo experiences many morphogenic movements simultaneously; changes in timing and/or amplitude of the various cellular mechanisms of one mechanism will 


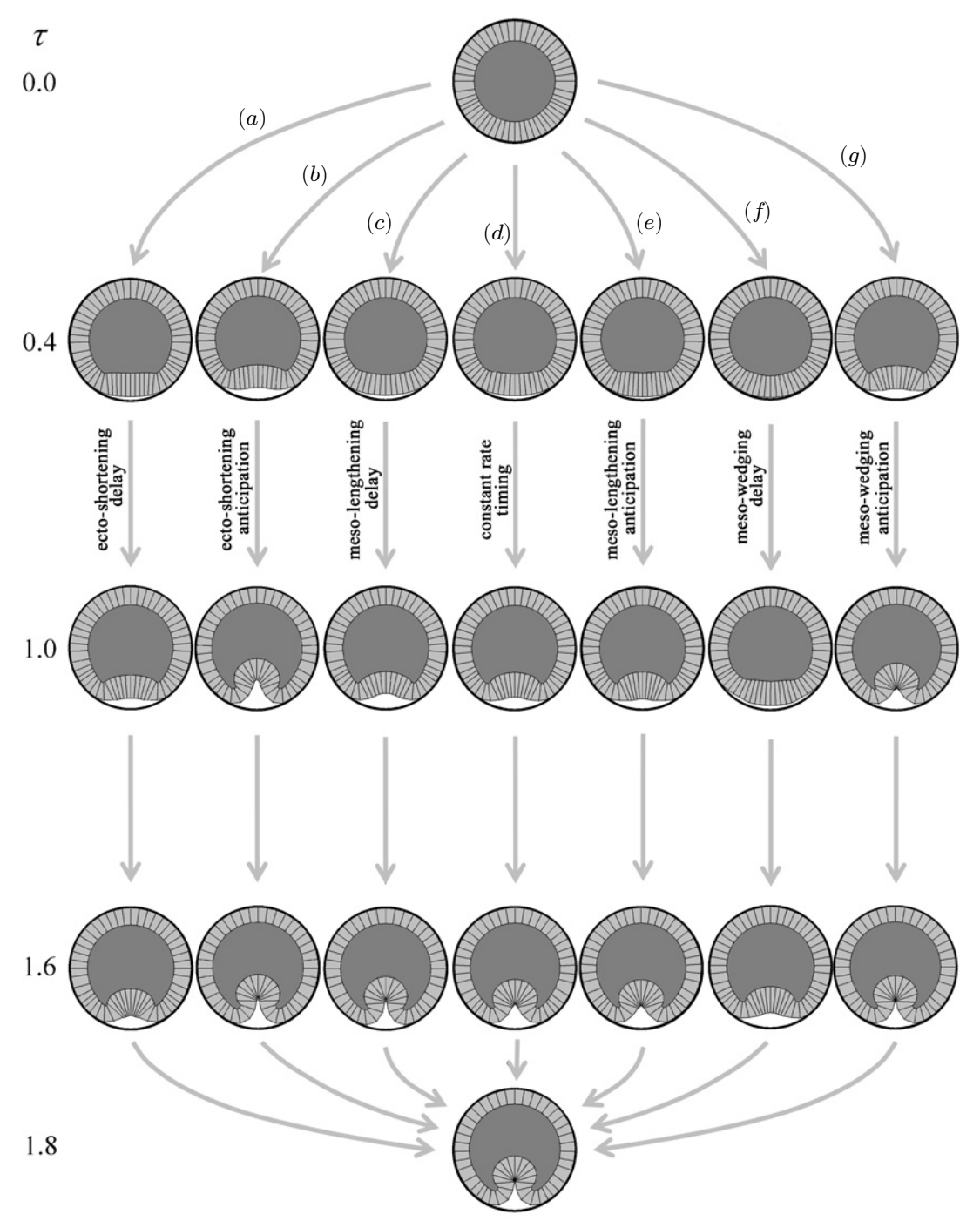

Figure 7. Schematic showing the effect of different invagination timing paths on intermediate and final phenotypes of the model for the simulation shown in figure 6 .

cause perturbations in the elastic force field of the embryo. Robustness to such perturbations is therefore of key importance to the survival of the embryo. Figure 9 shows that invaginated embryos with similar phenotypes can be obtained by combining three active shape changes in different ways, a phenomenon known as redundance, a phenomenon also previously studied by Clausi and Brodland (1993). The figure also shows that some invagination mechanisms are more robust to perturbations than others. Thus, should an invagination mechanism be partly impaired in one or several of its components, the action of a submechanism can compensate for that deficiency.

Consider, for instance, an invagination mechanism relying on a combination of mesodermal wedging and ectodermal pushing, a mechanism corresponding to the mid-point between node $\mathrm{B}$ and node $\mathrm{D}$ in the $\boldsymbol{\omega}$ sphere. Figure 9 provides a way to predict how the phenotype will be affected if specific active force generators are impaired. Loss or weakening of the mesodermal wedging due to mutation or environmental perturbation would push the phenotype towards node B, but still result in a successful invagination. The loss of ectodermal pushing would push the phenotype towards node $\mathrm{D}$, resulting in successful invagination, unless ectodermal pushing was completely lost. Likewise, the gaining of mesodermal lengthening through mutation or environmental perturbation would push the phenotype towards the $\overparen{\mathrm{DA}}$ arc resulting in at least partial invagination. The same is true of the gaining of mesodermal shortening, which would push the phenotype towards the $\overparen{\mathrm{DC}}$ arc resulting in at least partial invagination. The height of the furrow smoothly varies in this central part of the $\boldsymbol{\omega}$ sphere, see figure 10 , which means that perturbations in this area would not cause dramatic changes in the furrow geometry. Thus, it would clearly be to the organism's benefit from a robustness perspective to select invagination mechanisms combining a number of active shape changes and, so, for the wild type to situate itself in the middle of the spherical sector ABCD. Furthermore, such a plurality of different force regimens may be employed to different types of invagination in different species (Clausi and Brodland 1993). For instance, gastrulation in the honey bee (Roth 2004) closely mirrors phenotypes depicted in the central parts of the spherical sector $\mathrm{ABCD}$. 


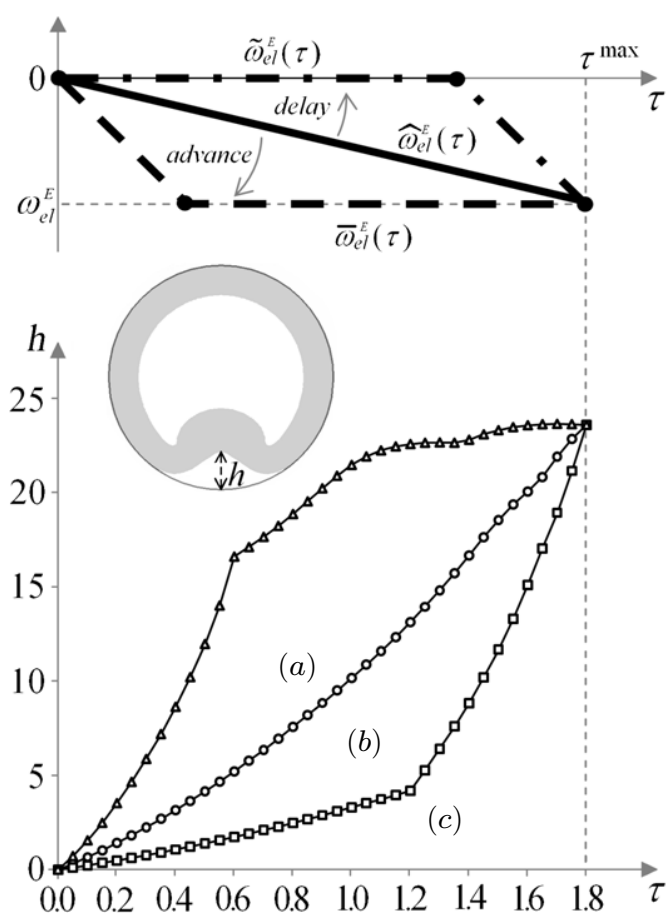

Figure 8. The temporal dependence of the quantifiable furrow parameter $h$ (below) corresponding to different ectodermal timing paths (above) in the case of the simulation shown in figure 6: (a) delay in ectodermal shortening onset $\tilde{\omega}_{e l}^{E}(\tau)$, see figure $7(a)$; (b) constant rate timing $\hat{\omega}_{e l}^{E}(\tau)$, see figure $7(d) ;(c)$ anticipation in ectodermal shortening timing $\bar{\omega}_{e l}^{E}(\tau)$, see figure $7(b)$.
What is also clear from figure 9 is that ectodermal pushing plays a very important role in invagination in this system. Without ectodermal pushing, no full invagination is possible, since along the mesodermal arcs, $\overparen{\mathrm{DA}}$ and $\overparen{\mathrm{DC}}$, there are no successful invaginating phenotypes and there is no complete invagination with only mesodermal shape changes. It is as we move towards the centre of the spherical sector ABCD, where the ectodermal pushing (node B) comes into play, that the majority of the successful invaginating phenotypes exist. Ectodermal pushing is so effective either as a component of an invagination mechanism or acting in isolation, that it seems highly likely that evolutionary pressure would favour it. Importantly, as this system analysis shows, ectodermal pushing does not prevent other invagination mechanics from operating in parallel.

A similar behaviour is shown by the hydrodynamic model of Pouille and Farge (2008), where the epithelial passive cell movements in response to the active apical constriction of mesodermal cells are sufficient to initiate the invagination process (Pouille and Farge 2008). In this 2D simulation a further radial centripetal force needs to be added in order to achieve the complete internalization of the furrow; such a force has been postulated to be due to the presence of the third dimension and, more specifically, to the anteriorposterior curvature of the 3D embryo (Pouille and Farge 2008). Similarly, in our model, passive epithelial movements in response to the apical constriction of the mesodermal cells are only able to form a shallow ventral furrow, as illustrated in

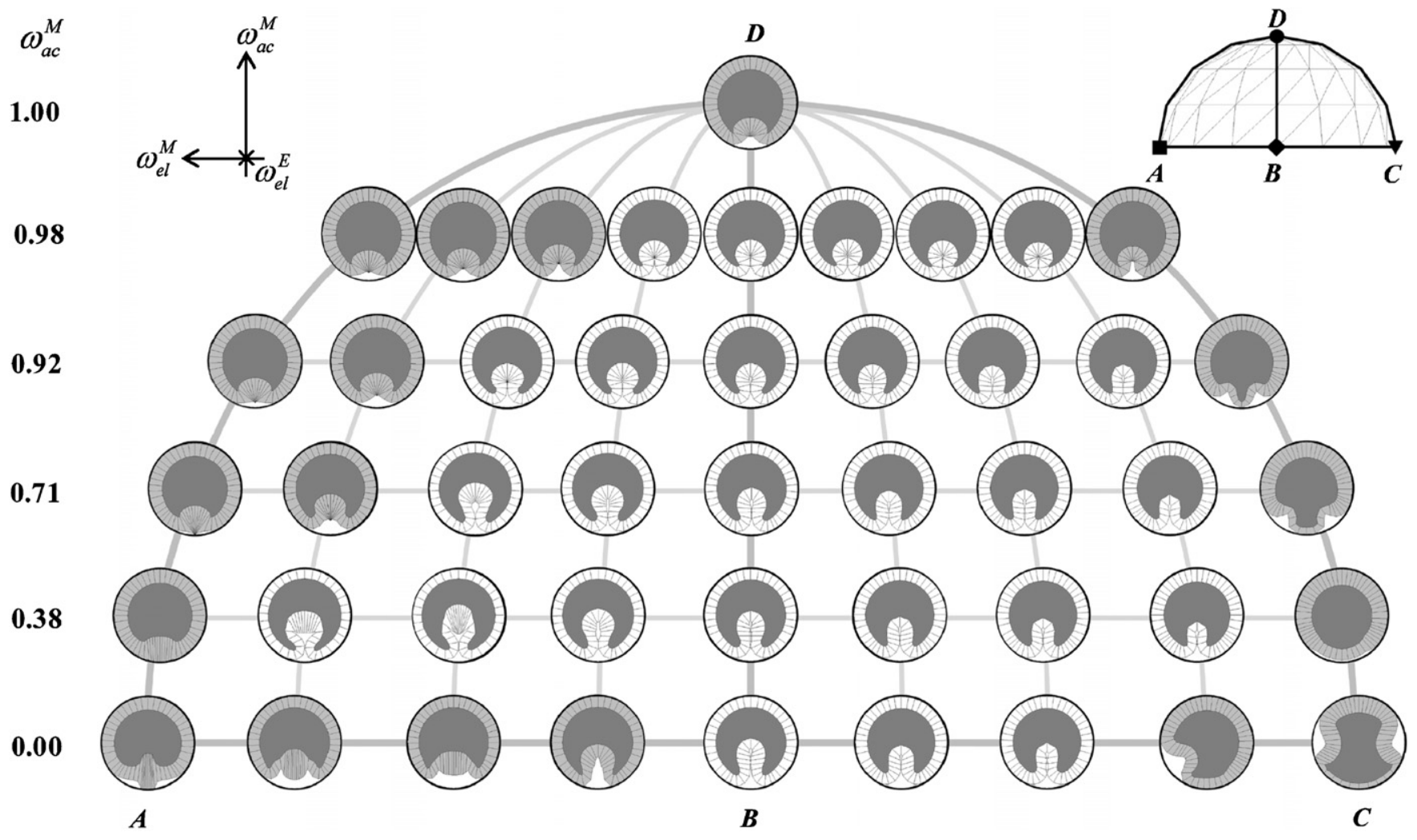

Figure 9. Phenotypes in the $\omega$ space: diagram of the final phenotypes corresponding to the vectors $\omega_{a}$ at reference nodal positions. 

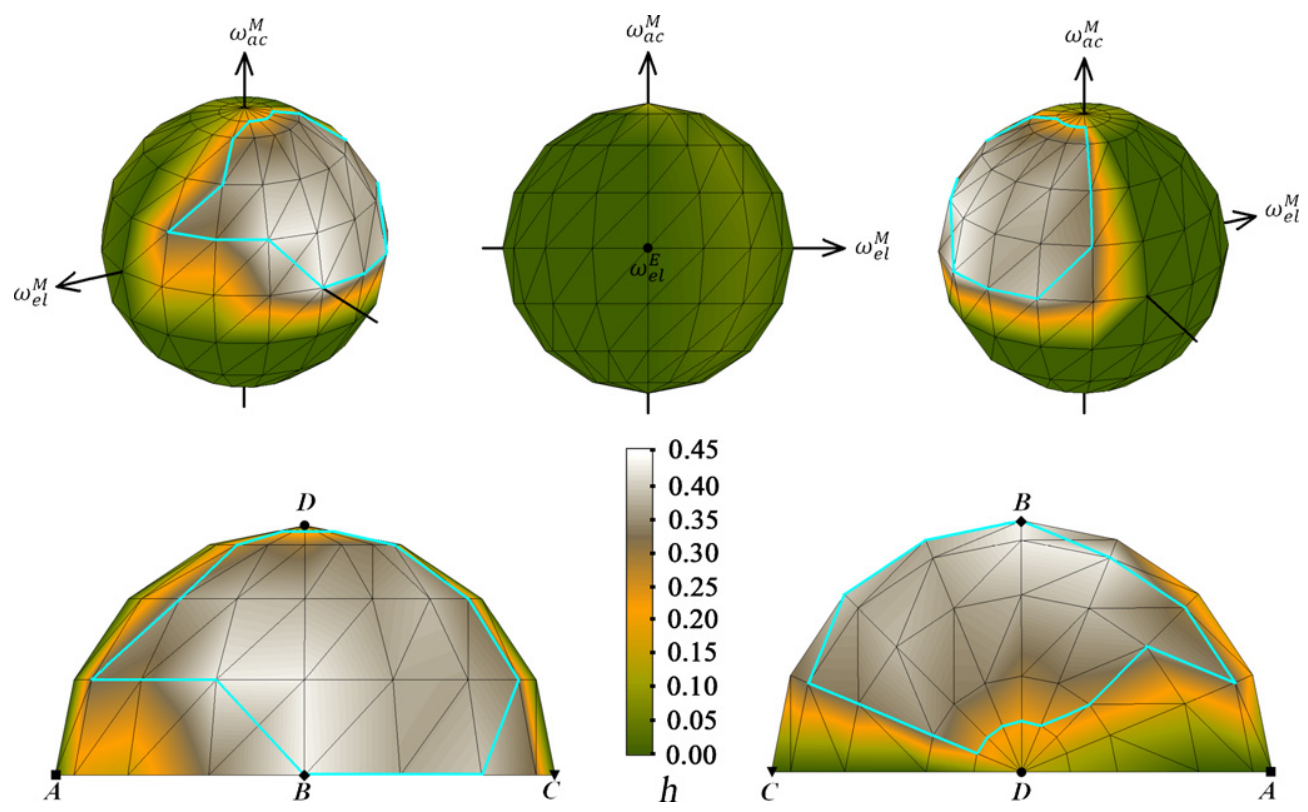

Figure 10. Spherical diagram for $h$ in the $\omega$ space: values of the quantity $h$ in the final phenotype corresponding to the generic vector $\omega_{a}$ are shown in different colours, in accordance with the key.

figure 9 by the phenotype corresponding to the point $D$ of the $\boldsymbol{\omega}$ sphere. Only by adding at least one other active force it is possible to achieve the rise and the complete internalization of the ventral furrow, as seen when ectodermal pushing is coupled to mesodermal wedging to obtain the invagination mechanism corresponding to the $\operatorname{arc} \widehat{\mathrm{BD}}$ (see figure 5).

Most experimental evidence in Drosophila points to a combination of active shape changes being responsible for invagination, although almost all exclude ectodermal pushing as a necessary component of invagination. A number of authors suggest that a contribution of apical constriction with apical-basal shortening in the mesoderm may be sufficient to generate invagination, on the grounds that mutants, which no longer have cells with an ectodermal identity, still form a ventral furrow (Leptin and Grunewald 1990, Costa et al 1993). However, as figure 9 shows, loss of ectodermal pushing still results in a partial furrow formation. Another argument against ectodermal pushing playing a significant role in ventral furrow formation in Drosophila is that if it were present, we might expect to be able to observe a defective furrow forming in the Twist-Snail double mutants. We point out, however, that if Snail suppresses ectodermal identity in the presumptive mesoderm, all cells will behave as ectoderm and push against one another. This corresponds to the condition along the $\overparen{\mathrm{BC}}$ arc, where we find invagination does indeed fail as mesodermal pushing counteracts the ectodermal pushing.

In Twist mutant embryos, mid-ventral cells still elongate to approximately the same length as they would in the wild type, even though they fail to undergo apical constriction or to undergo furrow formation, resulting in a thicker mesodermal primordium (Leptin and Grunewald 1990, Costa et al 1993). This implies that apical-basal cell lengthening in the mesodermal primordium is not simply a passive response to apical constriction (Costa et al 1993), as nuclei and cytoplasm are pushed basally. Significantly, ventral cells in Snail mutants exhibit the opposite behaviour. They shorten to form a thinner mesodermal primordium, even though they fail to undergo normal apical constriction and apical flattening (Leptin and Grunewald 1990); suggesting a Snail-independent role for Twist in cell shortening. When mesodermal shortening acts alone (node C), the results from the model mirror the Snail mutant phenotype, while the phenotypes of node A, node D and those along the $\overparen{\mathrm{DC}}$ arc, which display a thickening, and/or a shallow hollowing of the mesodermal primordium, concord with the Twist mutant phenotype (Leptin and Grunewald 1990, Costa et al 1993, Seher et al 2007). Mutants cnt48 which lack apical constriction are observed to develop no invagination but rather a thicker placode (Kolsch et al 2007), which correspond to phenotypes along the $\widehat{\mathrm{AD}}$ axis.

We should note that the shape of cells in the furrow in both wild type and mutant Drosophila melanogaster embryos may be influenced by other coincident morphogenetic movements (e.g. germ band extension, cephalic furrow and posterior midgut invaginations), either via changes in yolk pressure or by elastic stress fields within the epithelium. In the future, it will be important to investigate the impact of these complex effects, nevertheless ventral furrow formation proceeds relatively normally in animals with no A-P patterning.

Several deformation paths reveal a period of ventral flattening in the ventral domain, as has been described in vivo. Our analysis makes it clear that it is not necessary to invoke a separate mechanism to account for this, since ventral flattening can follow as an indirect consequence of the action of other forces. In the model, the timing of mesodermal lengthening has relatively little effect on the extent of ventral flattening. Instead, ventral flattening can be a passive response to apical constriction: its duration depending on the relative timing of other shape changes. We predict therefore that the extent of ventral flattening will mirror changes of invagination timing in Drosophila embryos. 
In this paper, we have used a mechanical model to investigate all the possible roles of three independent active cell shape changes in ventral furrow formation. In the present case and in contrast to other recent work (Taber 2008, Ramasubramanian and Taber 2008), we say nothing about the regulation of these shape changes, although we hope to do so in the future. The redundance of the invagination mechanisms (see figure 9) emphasizes that it is not possible to deduce the regimen of forces driving a particular morphogenetic event (Brodland and Clausi 1994) or to distinguish between active forces and the passive responses to these forces based on observation alone, e.g. using PIV studies (Supatto et al 2005). In order to do so, additional experimental data are needed. In this respect, by following an approach similar to that suggested in the work of Davidson et al (1995), in vivo experiments can be carried out to help to narrow the possibilities of those mechanisms that can produce similar phenotypes and to decide which mechanism really leads ventral furrow invagination. For instance, the determination of the experimental trends of the height, $h$, of the ventral furrow may help to decipher which are the main components determining the real mechanism of invagination. Resolving the invagination mechanism into constituent forces through genetic mutations will help to determine how specific cellular and molecular processes contribute to ventral furrow invagination (Davidson 2008).

Whether embryonic tissues behave as a viscous fluid or as an elastic solid, or both, during the slow process of morphogenesis is a matter still under debate, since relatively little data are available on the mechanical properties of embryonic tissues (Chen and Brodland 2008, Ramasubramanian and Taber 2008, Pouille and Farge 2008). The fluid behaviour is reflected in the viscous stresses (associated with the strain rates) and in the surface tension at the boundaries, while the aspects of solid behaviour (associated with strains) are indicated by the presence of permanent residual stresses (Forgacs et al 1998, Ramasubramanian and Taber 2008). It has been shown that some properties of certain living embryonic tissues can be explained by resorting to a viscoelastic model (Forgacs et al 1998) and that extending the viscous fluid analogy to cellular kinetics helps to interpret cell sorting (Beysens et al 2000) and cell rearrangement (Brodland and Veldhuis 2003). Furthermore, ventral invagination, which does not include changes in cell topology, has been modelled using a purely fluid behaviour of the epithelium in Pouille and Farge (2008). After neglecting convective terms and elastic forces and assuming that the cell boundaries are spherical, the authors obtain ventral invagination by modifying the apical membrane tension. In our case, the presence of the fluid is introduced as an incompressibility constraint, but our constitutive law is hyper elastic, which neglects the two main sets of forces encountered in fluids: viscous forces and surface tensions. The driving forces are given by the changes in the configuration of the cytoskeleton and could be validated by measuring the residual stresses of the epithelium. In the two types of simulations, by locally modifying either a material parameter of the fluid, or the microstructure of the solid, ventral invagination has been successfully achieved. It is likely that the correct constitutive laws for the passive component during Drosophila gastrulation combine both viscous and elastic responses, as modelled in amphibian gastrulation (Chen and Brodland 2008) or more generally in the fabric evolution of embryonic cells (Brodland and Veldhuis 2003, Brodland et al 2006). Whether the active component in Drosophila is associated with the fluid material parameters or with the structural rearrangements requires further experimental analysis.

Our assumption of hyper elasticity, although consistent with small morphogenetic time frames, is clearly at odds with studies of cells and tissues over longer time periods, where passive cells exhibit viscous components of deformation. Despite the fact that Drosophila invagination is very fast, it is important that the next refinement of our model should include these. Nevertheless, it is worth noting that the main interest of this work concerns the effect of combinations of shape changes driving the process of ventral furrow invagination; in this respect, the hypothesis does not allow us to investigate path dependence but does allow us to investigate robustness and redundancy - which is the focus of this paper.

Finally, our previous work concerning the development of a 3D Drosophila embryo's model showed that inclusion of the third dimension in the simulations increases the robustness of the $3 \mathrm{D}$ ventral furrow formation with respect to the $2 \mathrm{D}$ process, when the relative intensities of the driving forces of a prefixed invagination mechanism are perturbed (Conte et al 2008). Preliminary analyses carried out with the 3D model seem to confirm the importance of the ectodermal pushing activity attested by the 2D model; in fact, the removal of ectodermal pushing in the presence of movements involving active wedging and elongation of mesodermal cells is sufficient to invalidate the rise and the formation of a proper furrow in the 3D model (Conte et al 2008), as seen with the invagination mechanism corresponding to the arc $\overparen{\mathrm{AD}}$ (see figure 5). The complete analysis of the possible distinct invagination mechanisms leading 3D ventral furrow formation will be the object of future work, which will also enable us to investigate the redundancy and robustness to mutation of ventral furrow invagination in $3 \mathrm{D}$.

\section{Conclusion}

We have used a finite element model of ventral furrow formation to reveal that an epithelium may invaginate via several possible invagination mechanisms which are mechanically feasible in biological systems. This redundancy provides the embryo, as well as many other natural systems, with a robustness to mutations that, on an evolutionary scale, may partly impair the primary invagination mechanism. Therefore, invagination mechanisms involving several active shape changes are more robust to mutational and environmental perturbation. Our work shows that all of these possible invaginating mechanisms involve ectodermal pushing: this is significant and shows how important such a shape change can be in leading to ventral furrow invagination in Drosophila. 


\section{Acknowledgments}

The authors are grateful for useful discussions with Florian Ulrich and Eric Weischaus. JM is financially supported by the Spanish Research Program Juan de la Cierva, VC is supported by the Biological and Physical Sciences Research Council (BBRSC), BB is supported by the Royal Society and Cancer Research UK, MM is supported by King's College London. This support is greatly acknowledged.

\section{References}

Beysens D A, Forgacs G and Glazier J A 2000 Cell sorting is analogous to phase ordering in fluids Proc. Natl Acad. Sci. USA 97 9467-71

Brodland G, Chen D-L and Veldhuis J 2006 A cell-based constitutive model for embryonic epithelia and other planar aggregates of biological cells Int. J. Plast. 22 965-95

Brodland G and Veldhuis J 2003 A computer model for cell reshaping in epithelia due to in-plane deformation and annealing Comput. Methods Biomech. Biomed. Eng. 6 89-98

Brodland G W and Clausi D A 1994 Embryonic tissue morphogenesis modeled by FEM J. Biomech. Eng. 116 146-55

Chen D-L and Brodland G W 2008 Multi-scale nite element modeling allows the mechanics of amphibian neurulation to be elucidated Phys. Biol. 5 1-15

Clausi D A and Brodland G W 1993 Mechanical evaluation of theories of neurolation using computer simulations Development 118 1013-23

Conte V, Muñoz J J and Miodownik M 2008 3D finite element model of ventral furrow invagination in the Drosophila melanogaster embryo J. Mech. Behav. Biomed. Mater. 2 188-98

Costa M, Sweeton D and Wieschaus E 1993 The Development of Drosophila Melanogaster (New York: Cold Spring Harbor Laboratory Press) chapter 8

Davidson L A 2008 Apoptosis turbocharges epithelial morphogenesis Science 321 1641-2

Davidson L A, Koehl M A R, Keller R and Oster G F 1995 How do sea urchins invaginate? Using biomechanics to distinguish between mechanisms of primary invagination Development $1212005-18$

Davidson L A, Oster G F, Keller R and Koehl M A R 1999 Measurements of mechanical properties of the Blastula wall reveal which hypothesized mechanisms of primary invagination are physically plausible in the sea urchin Strongylocentrotus purpuratus Dev. Biol. 209 221-38

Forgacs G, Foty R A, Shafrir Y and Steinberg M S 1998 Viscoelastic properties of living embryonic tissues: a quantitative study Biophys. J. 74 2227-34
Keller R, Davidson L A and Shook D R 2003 How we are shaped: the biomechanics of gastrulation Development 71 171-205

Kolsch V, Seher T, Fernandez-Ballester G J, Serrano L and Leptin M 2007 Control of Drosophila gastrulation by apical localization of adherens junctions and RhoGEF2 Science 132 384-6

Lecuit T and Lenne P F 2007 Cell surface mechanics and the control of cell shape, tissue patterns and morphogenesis Nature Rev. 8 633-44

Leptin M 1995 Drosophila gastrulation: from pattern formation to morphogenesis Annu. Rev. Cell Dev. Biol. 11 189-212

Leptin M 1999 Gastrulation in Drosophila: the logic and the cellular mechanisms EMBO J. 18 3187-92

Leptin M and Grunewald B 1990 Cell shape changes during gastrulation in Drosophila Development 110 72-84

Muñoz J, Barrett K and Miodownik M 2007 A deformation gradient decomposition method for the analysis of the mechanics of morphogenesis J. Biomech. 40 1372-80

Muñoz J J 2008 Modelling unilateral frictionless contact using the null-space method and cubic B-spline interpolation Comput. Meth. Appl. Mech. Eng. 197 979-93

Muñoz J J and Jelenić G 2004 Sliding contact conditions using the master-slave approach with application on the geometracally nonlinear beams Int. J. Solids Struct. 41 6963-92

Odell G M, Oster G, Alberch P and Burnside B 1981 The mechanical basis of morphogenesis: I. Epithelial folding and invagination Dev. Biol. 85 446-62

Pouille P A and Farge E 2008 Hydrodynamic simulation of multicellular embryo invagination Phys. Biol. 5015005

Ramasubramanian A and Taber L A 2008 Computational modeling of morphogenesis regulated by mechanical feedback Biomech. Model. Mechanobiol. 7 77-91

Rodriguez E K, Hoger A and McCulloch A D 1994 Stressdependent finite growth in soft elastic tissues J. Biomech. 27 455-67

Roth S 2004 Gastrulation Gastrulation in Other Insects (New York: Cold Spring Harbor Laboratory Press) chapter 8, pp 105-121

Seher T C, Narasimha M, Vogelsang E and Leptin M 2007 Analysis and reconstitution of the genetic cascade controlling early mesoderm morphogenesis in the Drosophila embryo Mech. Dev. 124 167-79

Supatto W, Débarre D, Moulia B, Brouzés E, Martin J L, Farge E and Beaurepaire E 2005 In vivo modulation of morphogenetic movements in Drosophila embryos with femtosecond laser pulses Proc. Natl Acad. Sci. USA 102 1047-52

Sweeton D, Parks S, Costa M and Wieschaus E 1991 Gastrulation in Drosphila: the formation of the ventral furrow and posterior midgut invaginations Development 112 775-89

Taber L A 2008 Theoretical study of beloussovs hyper-restoration hypothesis for mechanical regulation of morphogenesis Biomech. Model. Mechanobiol. doi: 10.1007/s10237-007-0106-x 